\title{
Evidence for the class of the most luminous quasars
}

\section{Linear sizes and the core-size relation of double radio sources}

\author{
P. Teerikorpi ${ }^{\star}$
}

Tuorla Observatory, University of Turku, 21500 Piikkiö, Finland

Received 16 May 2000 / Accepted 30 May 2001

\begin{abstract}
We study the radio properties of the optically luminous "AI" subclass of quasars at $M_{V} \approx-26.0$ $\left(H_{0}=100 \mathrm{~km} \mathrm{~s}^{-1} / \mathrm{Mpc}, q_{0}=\frac{1}{2}\right.$; Teerikorpi 1981, 2000; Papers I, II), after first results on double sources in Paper I and the confirmation in Paper II that optical activity abruptly increases when one crosses the gap around $M_{V}=-25.5$ between AI and the optically fainter quasars. AI double sources define the upper envelope in the size vs. redshift diagram, so AI quasars are not only optically the most luminous, but also in radio the largest objects in their redshift range (from 0.5 up to at least 1.6). They are typically good FRII objects, mostly with a small lobe-distance asymmetry $Q$. A part of the scatter in size is due to orientation, which reveals itself via the size vs. core strength dependence, such as is expected from relativistic core boosting with $\Gamma \approx 5-8$. This relation appears for symmetric sources $(Q<1.5)$ and especially clearly for sources with lobe spectral indices either shallow or steep ("Kardashev"). AI sources occupy the upper envelope of the latter core-size relation. Asymmetric sources $(Q \geq 1.5)$ or those with intermediate lobe spectral indices $0.7 \leq \alpha_{\mathrm{L}} \leq 1.0$ do not show any core-size dependence. Their bending angle does not converge towards a small value for large sizes, as it does for the sources with a strong core-size relation. The data suggest (as in Paper I) that the asymmetry $Q$ increases from AI to the edge of the fainter population, which, down to $M_{V} \approx-24.8$, contains unusual double sources, with a more versatile and less symmetric lobe structure, often with a hotspot in the trailing edge.
\end{abstract}

Key words. quasars: general

\section{Introduction}

The present paper continues to study the properties of the optically luminous subclass of radio loud quasars, separated by a gap in the absolute magnitude distribution from fainter quasars. This gap at $M_{V} \approx-25.5$ was suggested in Paper I (Teerikorpi 1981). Confirmed in Paper II (Teerikorpi 2000) ${ }^{1}$, it appears at least within the redshifts $0.5-1.6$. Below $z \approx 0.5$ the quasars on the bright side are rare. Paper I noted a change in optical variability across the gap: the luminous quasars around $M_{V}=-26.0$ (termed "AI" in Paper II) are less active than those on the fainter side of the gap. This was confirmed by new data in Paper II, including optical polarization.

Paper I also discussed the radio properties and concluded: "When they [AI] are double sources, or triple sources with small central fluxes, their radio structure is more symmetric than that of their counterparts at the

\footnotetext{
* e-mail: pekkatee@astro.utu.fi

1 We still use the archaic value $H_{0}=100 \mathrm{~km} \mathrm{~s}^{-1} / \mathrm{Mpc}$ for the Hubble constant, as was done in Papers I and II $\left(q_{0}=\frac{1}{2}\right)$.
}

fainter side of the gap, and they may resemble more radio galaxies in their radio properties".

Here we study, using the more extensive data now available, which of the double source properties differ across the optical gap. We examine in particular the size and the size vs. core strength dependence. Are the AI among the first-ranked quasars also in radio size?

\section{Data for double radio sources}

Our basic source is Nilsson (1998), containing 1038 double sources (365 are quasars). Table A.1 shows data for the Paper II quasars $\left(-26.5<M_{\min }<-23.8\right)$ with known double structure: redshift $z, \mathrm{~K}$ - and extinction corrected minimum level absolute $V$-mag $M_{\text {min }}$, angular size LAS, flux $S 5 \&$ core flux $S 5_{\mathrm{c}}$ at $5 \mathrm{GHz}$, bending angle $\Phi$, spectral index $\alpha_{2.7-5.0}$, asymmetry $Q$, flux asymmetry $f_{\mathrm{r}}$, derived lobe spectral index $\alpha_{\mathrm{L}},(\log )$ size $\log D, \mathrm{~K}$-corrected core $(\log )$ power $\log P_{\mathrm{C}}$ at $5 \mathrm{GHz}^{2}$.

\footnotetext{
2 In the diagrams, we give power without a constant factor: $P_{5 \mathrm{GHz}}=(z+1-\sqrt{1+z})^{2}(1+z)^{\alpha-1} f_{5 \mathrm{GHz}}$, where $f_{\nu} \propto \nu^{-\alpha}$.
} 

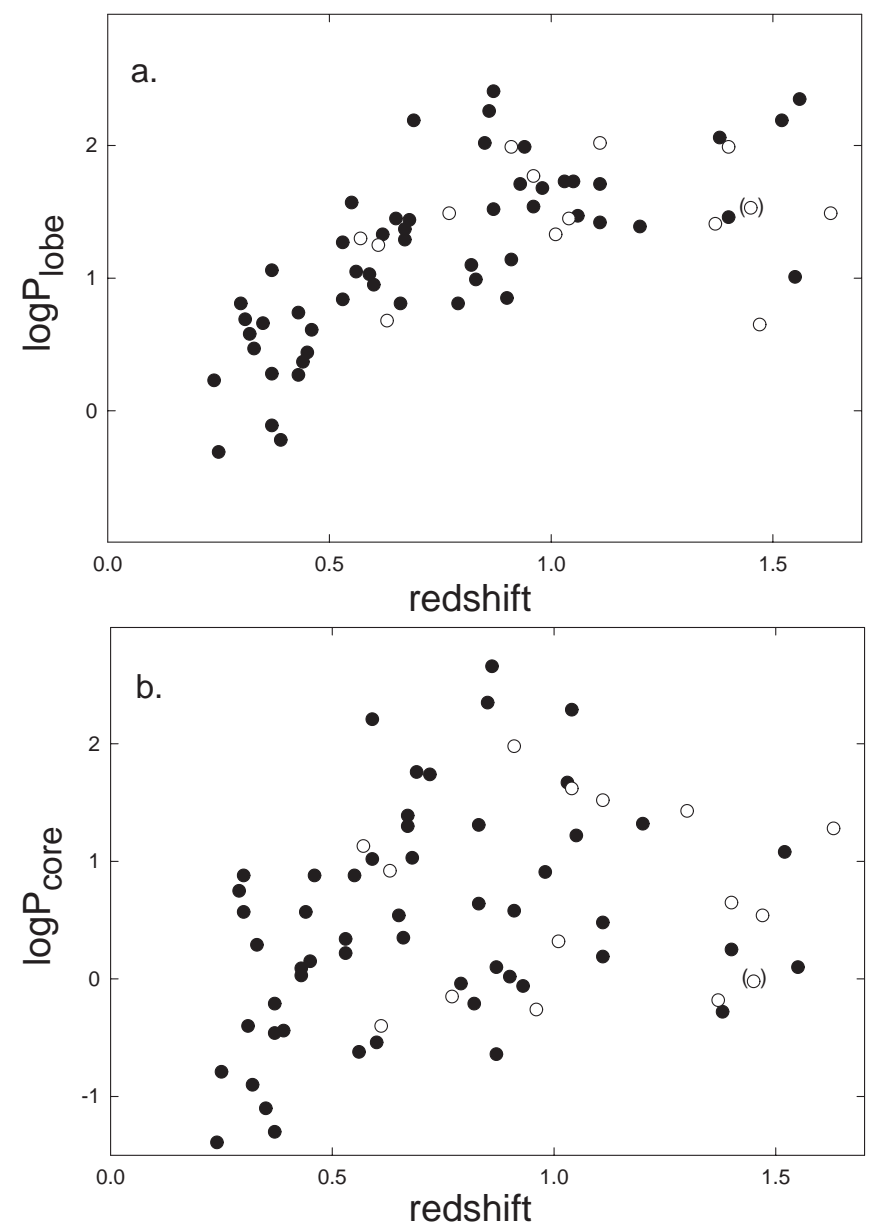

Fig. 1. a) Lobe luminosity $\log P_{\text {lobe }}$ versus redshift $z$, and b) core power $\log P_{\text {core }}$ versus $z$. Open circle: AI.

Core flux and, hence, spectral index may change in observable time--scales. This also affects the lobe spectral index derived from the total flux spectral index and coreto-lobe flux ratio.

We have calculated the lobe spectral index $\alpha_{\mathrm{L}}$ in Table A1 as follows. We subtract the core from the total flux and solve the lobe index between 2.7 and $5 \mathrm{GHz}$ from:

$\alpha_{\mathrm{L}}=\alpha_{2.7-5.0}+1.62\left[\ln (1+q)-\ln \left(1+q(5 / 2.7)^{\alpha_{\mathrm{C}}-\alpha_{\mathrm{L}}}\right)\right]$

where $q=$ observed core-to-lobe flux ratio at $5 \mathrm{GHz}$ $(1.62=1 / \ln (5 / 2.7))$. A core spectral index $\alpha_{\mathrm{C}}=0.2$ was assumed (Lister et al. 1994; Valtaoja et al. 1988). For the few undetected cores, we put the flux equal to zero.

The uncertainty in the derived $\alpha_{\mathrm{L}}$ is about \pm 0.05 , when the core flux varies \pm 30 percent, $\alpha_{\mathrm{C}}$ ranges from $0.0-0.2$, and $q \approx 0.2$. For $q \approx 0.4$, the error in $\alpha_{\mathrm{L}}$ may be \pm 0.1 . Limiting to the core-to-total flux ratio $<0.9$ excludes very uncertain values of $\alpha_{\mathrm{L}}$.

\section{Lobe and core powers versus $z$}

The present data are not well suited to determine how the radio luminosity changes from AI to fainter quasars because of the usual strong flux selection.
Nevertheless, from Fig. 1a one may conclude that the maximal lobe luminosities are not very different for these subsamples ${ }^{3}$. In the core power vs. redshift diagram (Fig. 1b) one would be especially interested in the lower envelope, where one expects to approach the little-boosted core strengths. There again the AI do not differ from the other quasars. However, Fig. 1b suggests that small core fluxes are missing from the sample.

As more distant radio sources are stronger, and there is evidence for distorted morphology in high- $z$ quasars (Barthel \& Miley 1988), one must keep in mind that some differences between subsamples may be due to redshift dependent properties. As in Paper II, we generally restrict the $z$ range to $0.5-1.65$. AI is absent at smaller $z$.

\section{Linear size and core power}

As the linear size depends on redshift, it is appropriate to compare the sizes of AI and fainter quasars by inspecting the size vs. redshift diagram. Figure 2a shows that AI double sources define the upper envelope in this diagram, hence they are extreme objects not only in their optical luminosity, but also in their radio size in the redshift range where they are found ${ }^{4}$. The scatter downwards is partly due to sources with strong cores (Fig. 2b), suggesting a viewing angle effect. Note that in Fig. 2b, 7 out of 9 (8) AI sources are found above or close to the upper envelope for the fainter quasars.

If one considers the whole sample in the range $0.5<z<1.65$, there is only a weak sign of a core strengthsize dependence. However, asymmetry or, in particular, lobe spectral index allows one to select a subsample where the core strength seems to be a good orientation indicator.

\subsection{Quasars with low and high asymmetry}

The asymmetry $Q$ is the ratio of lobe distances from the core (by definition $>1$ ). Figure 3 shows the size vs. core strength diagram for symmetric $(Q<1.5)$ and asymmetric $(Q \geq 1.5)$ sources. The former reveals a clear trend, while for the more asymmetric sources there is no correlation.

\subsection{Quasars with steep or shallow lobe spectrum}

The quasars with high asymmetry are concentrated in the spectral range $0.7 \leq \alpha_{\mathrm{L}} \leq 1.0$ (Fig. 4). Now using the spectral index $\alpha_{\mathrm{L}}$ instead of $Q$ to divide the sample, we

\footnotetext{
${ }^{3}$ In Paper II the gap separating AI from the fainter quasars, was best seen with the formalism of the cosmological Malmquist bias. The position of $0856+1703$ in the $m-\Delta \log z$ diagram did not allow one to decide to which side of the gap it belongs (in Fig. 5 of Paper II, the dot close to the right end of the gap). With $M_{\min }=-25.66$, we display this quasar as bracketed.

4 The linear size is derived for $q_{0}=0.5$ and $h=1$ as $D / \mathrm{kpc}=$ $1000 \operatorname{LAS}\left(6000 / 206265^{\prime \prime}\right)(\sqrt{1+z)}-1) /(1+z)^{3 / 2}$.
} 

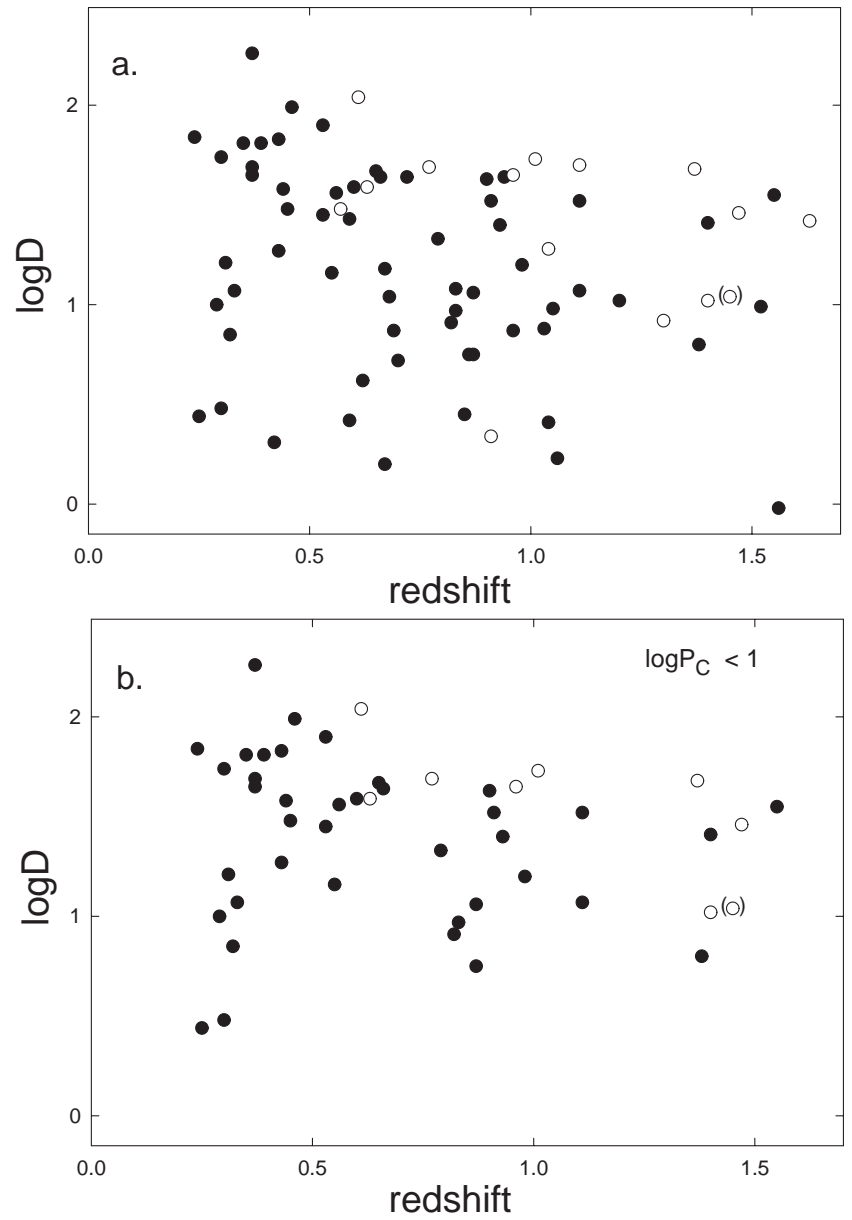

Fig. 2. Linear size $\log D$ against redshift $z$ a) for the whole sample. b) for quasars with $(\log )$ core power $<1$. Open circle: AI.

find that outside of this range (i.e. for shallow and steep spectra) there is a quite good correlation between size and core power, as displayed in Fig. 5a (the steep and shallow spectra are rather similarly distributed; not shown).

The correlation is not due to the redshift dependence of both size and core luminosity: it is also seen for pairs with similar redshifts. Within the twin beam theory of double sources, it is in the sense of core Doppler boosting, when the viewing angle decreases (Orr \& Browne 1982). See Sect. 6 for the required Lorentz factor $\Gamma$. For a narrow optical range, the core strength has the role of the orientation indicator " $R_{V}$ " (= radio core power/optical luminosity; Wills \& Brotherton 1995; Barthel et al. 2000).

Now AI sources with large viewing angles form a nice upper envelope (Fig. 5a). This could mean either that 1) intrinsic core strengths (jets) are similar and AI are larger in size, or 2) the sizes are similar, but in AI the jets are stronger.

The first alternative is perhaps favoured by the $z$-size diagram (Fig. 2). In the second case, the shift of AI is in the sense of the Wills-Brotherton (1995) conjecture (intrinsic jet $\propto$ optical power). The true situation may be somewhere between the two. Figure 9 in Sect. 6 shows the diagram after the Wills \& Brotherton correction,
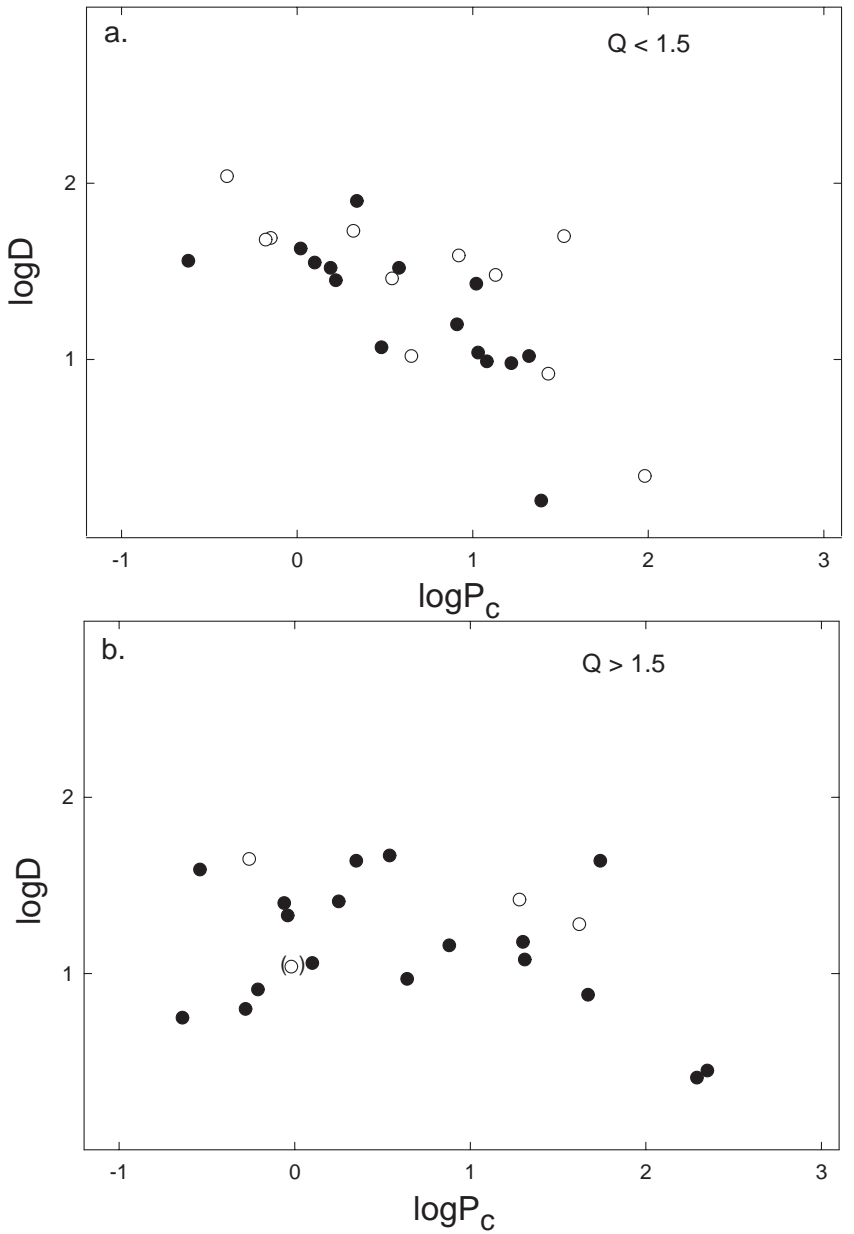

Fig. 3. Linear size $\log D$ vs. core power $\log P_{\mathrm{C}}$, for sources with a) small asymmetry $(Q<1.5)$ and b) high asymmetry $(Q \geq 1.5)$. $P_{\mathrm{C}}$ is defined in Sect. 2. Open circle: AI.

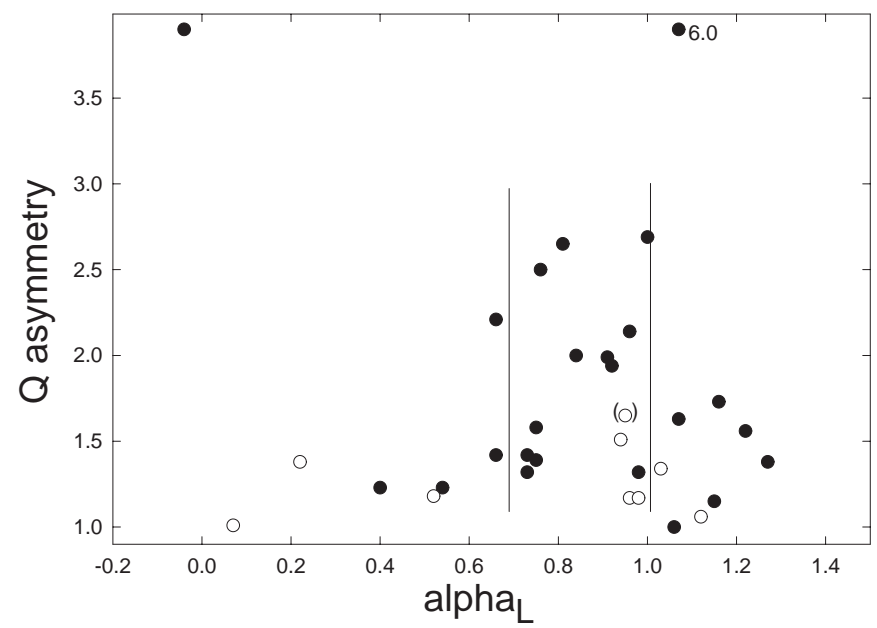

Fig. 4. Asymmetry $Q$ vs. spectral index $\alpha_{\mathrm{L}}$ for weak core sources $\left(\log P_{\mathrm{C}}<1.0\right)$. Open circle: AI.

normalizing the core power to a constant optical luminosity: $\log P_{\mathrm{n}}=\log P_{\mathrm{c}}+0.4\left(M_{\min }-(-25.0)\right)$. It is interesting that generally the fainter quasars shift close to the same line with the AI, except for a few sources (those within the magnitude range $-25.5--24.8$, shown as open 

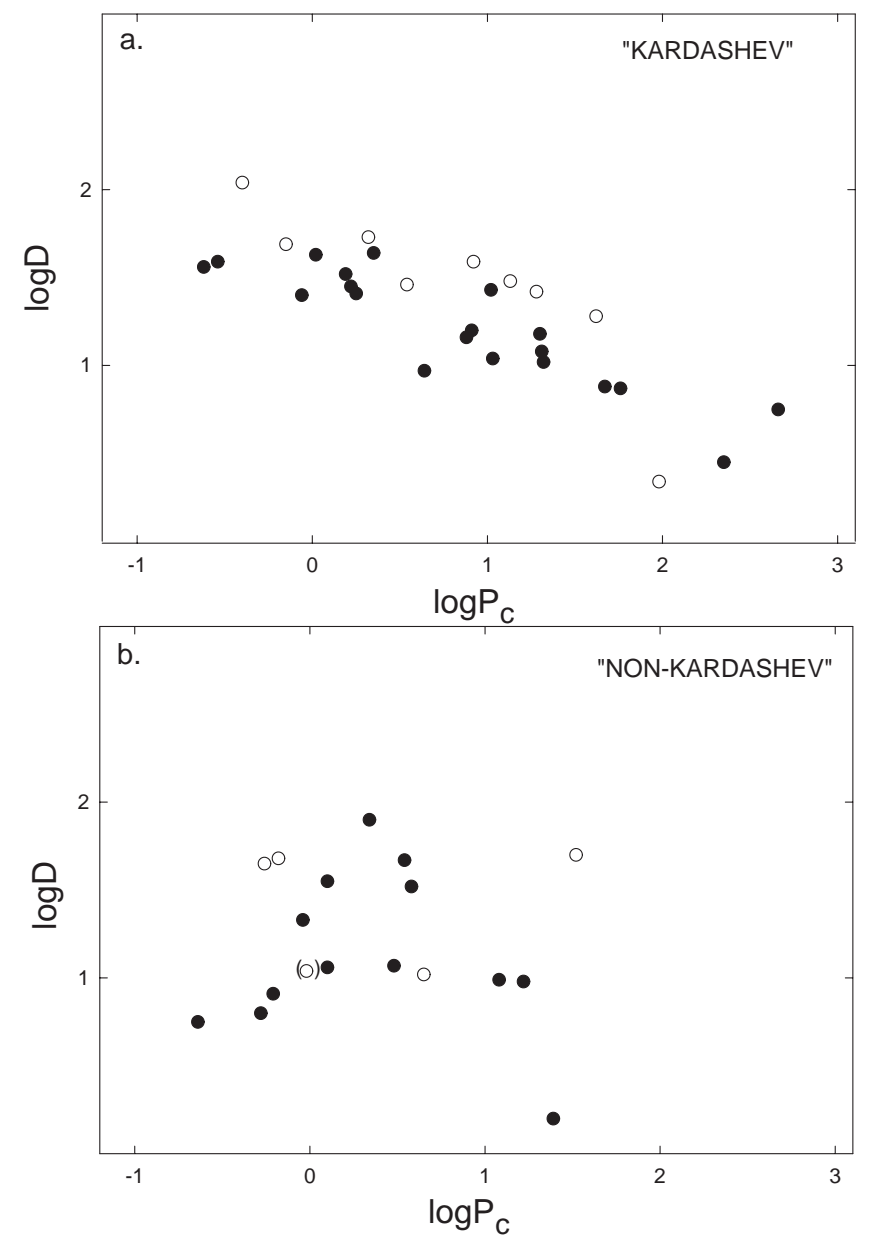

Fig. 5. Linear size $\log D$ vs. core power $\log P_{\mathrm{C}}$. when a) $\alpha_{\mathrm{L}}<$ 0.7 or $\alpha_{\mathrm{L}}>1.0$, and $\left.\mathbf{b}\right) 0.7 \leq \alpha_{\mathrm{L}} \leq 1.0$. Open circles: AI.

circles; cf. Sect. 5) which remain below the main body of the normalized relation.

\subsection{Quasars with intermediate spectral index}

On the contrary, the sources in the range $0.7 \leq \alpha_{\mathrm{L}} \leq 1.0$ show quite a scattered distribution on the core power-size diagram (Fig. 5b). Most of these sources are not good FRII types, and are often quite asymmetric in $Q$.

\subsection{Kardashev effect?}

The appearance of the core-size relation for shallow and steep spectra brings to mind the Kardashev (1962) effect which makes one suppose that such sources are enjoying a continuous stream of jet energy, which keeps them for a long time in that special phase of energy loss. Then the observed core power would reflect the (boosted) strength of this jet and the good core- size relation may be interpreted as an orientation effect plus relativistic core boosting ${ }^{5}$.

\footnotetext{
${ }^{5}$ Following, through a fixed frequency window, the growth of a synchrotron source, one would measure a range of spectral indices, from negative ones ( $\alpha<0$; self-absorption) through a flat phase (0.0-0.5) to steep ones $(\alpha>1$; energy losses). In
}
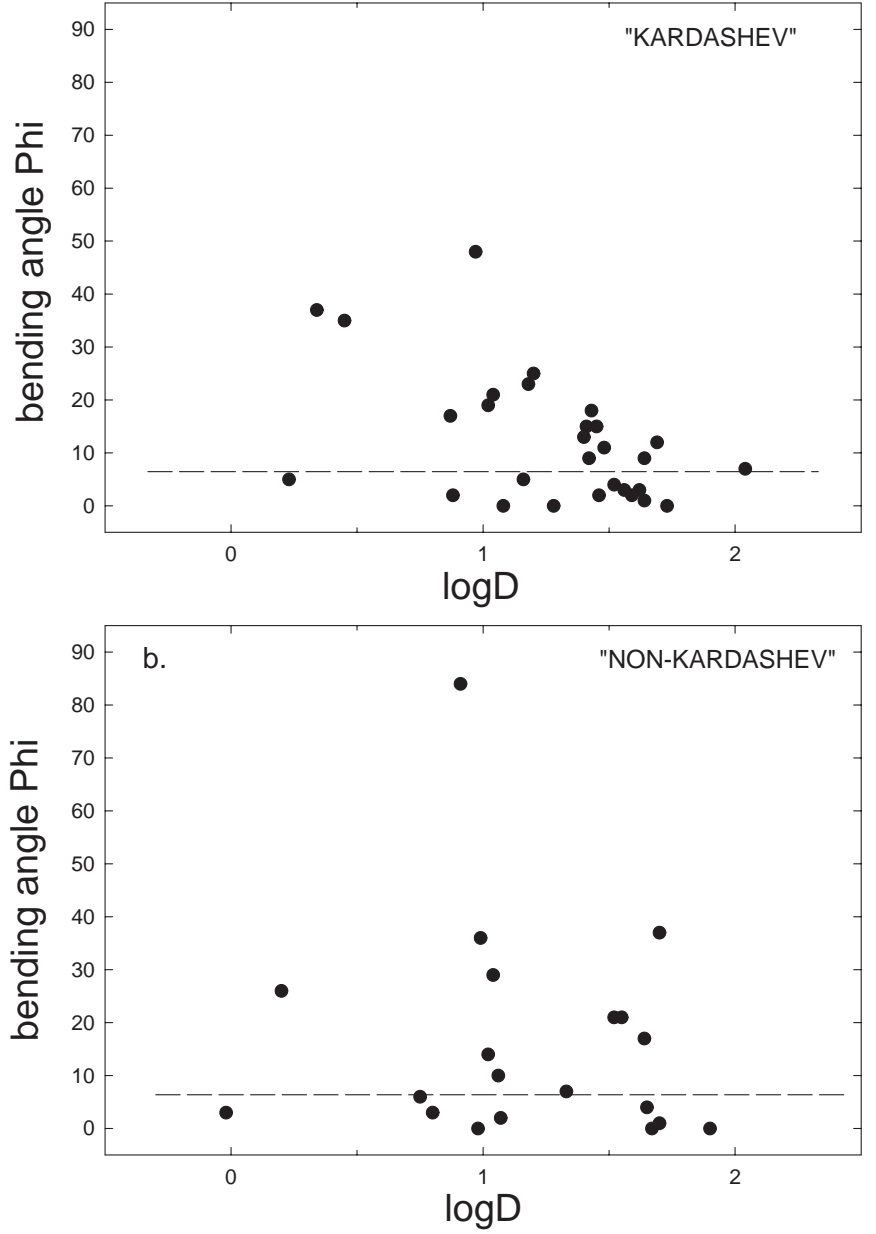

Fig. 6. Bending angle $\Phi$ vs. linear size $\log D$ a) when $\alpha_{\mathrm{L}}<0.7$ or $\alpha_{\mathrm{L}}>1.0$, and $\mathbf{b}$ ) when $0.7 \leq \alpha_{\mathrm{L}} \leq 1.0$. Redshifts are $0.5<z<1.65$. The line indicates $\Phi=6^{\mathrm{o}}$.

One may think that the intermediate spectral index range is underpopulated by "good" doubles which spend longer times on both sides of the Kardashev spectral break. A concentration of indexes in this range could occur for doubles which are disturbed away from a symmetrical Kardashev process. In fact, in this $\alpha_{\mathrm{L}}$ range a majority of the sources have $Q \geq 1.5$ (Fig. 4).

\subsection{The bending angle}

Figure 6 shows the angle $\Phi$ against (log) size. The bending angle of "Kardashev" sources converges to a small value at large size. This is as expected if their apparent size is a measure of orientation. At small viewing angles, intrinsic deviations from the source axis are amplified into larger observed misalignment (Baryshev \& Teerikorpi 1995).

the continuous ejection model (Kardashev 1962), steepening happens via the shift of the break frequency $f_{\mathrm{T}}$ where the index changes by $\Delta \alpha=0.5$. For hotspots, Meisenheimer et al. (1989) found that $\alpha \approx 0.5$ below the break and $\approx 1.0$ above it. 


\section{Possible changes across the optical gap}

The above results suggest that AI double sources are larger than those on the faint side of the optical gap. In Paper I there was an indication that the asymmetry $Q$ increases abruptly, as if the edge of the fainter population already contained objects differing from those in AI. Paper II gave evidence that optical activity increases abruptly from AI to the fainter edge. Here we consider $Q$, $\alpha_{\mathrm{L}}$, and source morphology. The treatment is brief and tentative, as going towards optically fainter quasars also means a shift in average redshift, which may result in spurious effects. Nevertheless, we feel that it is warranted to point out possible changes across the optical gap.

\subsection{Asymmetry $Q$ in core-lobe distances}

In sources with weak cores, $Q$ was seen to increase abruptly when one crosses the gap between AI and the fainter quasars (Paper I). The new data also suggest such a trend, as seen for $S_{\text {core }} / S_{\text {lobe }}<0.3$ (Fig. 7a). When stronger cores are allowed, the scatter increases.

To compare AI and the fainter quasars, one would preferably have about equal numbers of objects, starting from the faint edge of the gap $\left(N_{\mathrm{AI}}=N_{\text {edge }}\right)$. As the latter are in any case more numerous, we allow different depths $N_{\text {edge }}=N_{\mathrm{AI}}+k \sigma_{N}$, where $\sigma_{N}=\sqrt{N_{\mathrm{AI}}}$, and $k=0,1,2$. Another choice would be to cover similar ranges in $M$, meaning (-25.5--24.8), as AI occupies (-26.3--25.5). Such choices keep the redshift difference small. Further extension of the range of faint quasars down to -23.8 causes a large shift in $\langle z\rangle$.

Applying the median test (e.g. Mack 1975) to the $Q$ distributions in Fig. 7a shows that the probabilities that the medians of $\mathrm{AI}$ and the first $N_{\mathrm{AI}}+k \sigma_{N}$ fainter quasars come from the same distribution are less than $0.25,0.05$, and 0.25 for $k=0,1,2$, respectively. Without $0856+1703$, these are $0.05,0.05,0.05$. For all sources (Fig. 7b) the probabilities are smaller (the increased scatter is compensated for by the larger numbers): 0.05, 0.05, 0.05, and 0.01, $0.025,0.025$. Thus the new data lend some support to the result in Paper I, where the sample was smaller and the asymmetry referred to average lobes, while now $Q$ mostly was calculated by Nilsson (1998) from resolved outermost hotspots.

Such a behaviour of $Q$ might be understood in the light of core boosting. When the core is weak, one looks at the source with a large viewing angle $\phi$ ("from the side") and sees intrinsic asymmetries and their differences. When $\phi$ is small (the core is strong), asymmetries are either enhanced or diminished, by projection and finite light speed (Baryshev \& Teerikorpi 1995).

\subsection{Lobe spectral index}

Figure 8 shows the derived lobe index $\alpha_{\mathrm{L}}$ vs. $M_{\min }$ for $f_{\mathrm{c}}<0.9$. Within $-25.5--24.8$ the indices cluster between

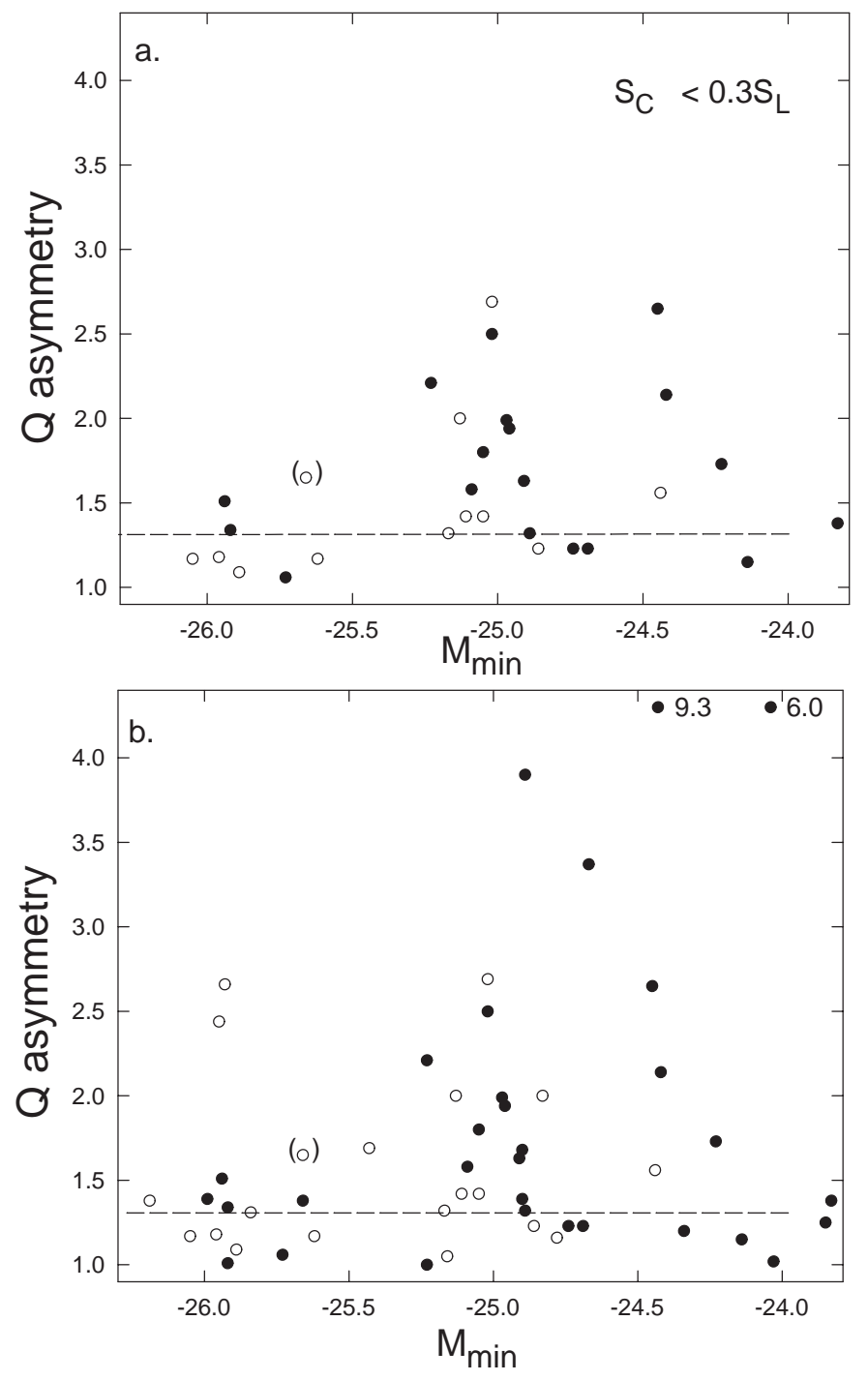

Fig. 7. Asymmetry $Q$ vs. $M_{\min }$, for $M_{\min }<-24.8$ a) when core flux/lobe flux $<0.3, \mathbf{b})$ for all sources. The line is the median of $Q$ for all AI. Dots are from the low redshift range $0.5-1.0$, circles refer to $1.0-1.65$.

0.7 and 1.0, while AI show a concentration around 1 , and a group of sources below $\alpha \approx 0.6^{6}$.

However, such a visual impression that the distributions of the spectral index on either side of the gap differ, is only mildly supported by the non-parametric "run test" (sensitive to location and dispersion; see Mack 1975): the probability that the two distributions come from the same population is less than 10 percent for the depth $k=1$, but higher for $k=0.2$.

\footnotetext{
${ }^{6}$ In fact, it was this diagram that suggested in the first place that the lobe spectral index $\alpha_{\mathrm{L}}$ might be an important parameter for the present study and led us to inspect its connections with asymmetry and the core-size relation (Sect. 4).
} 


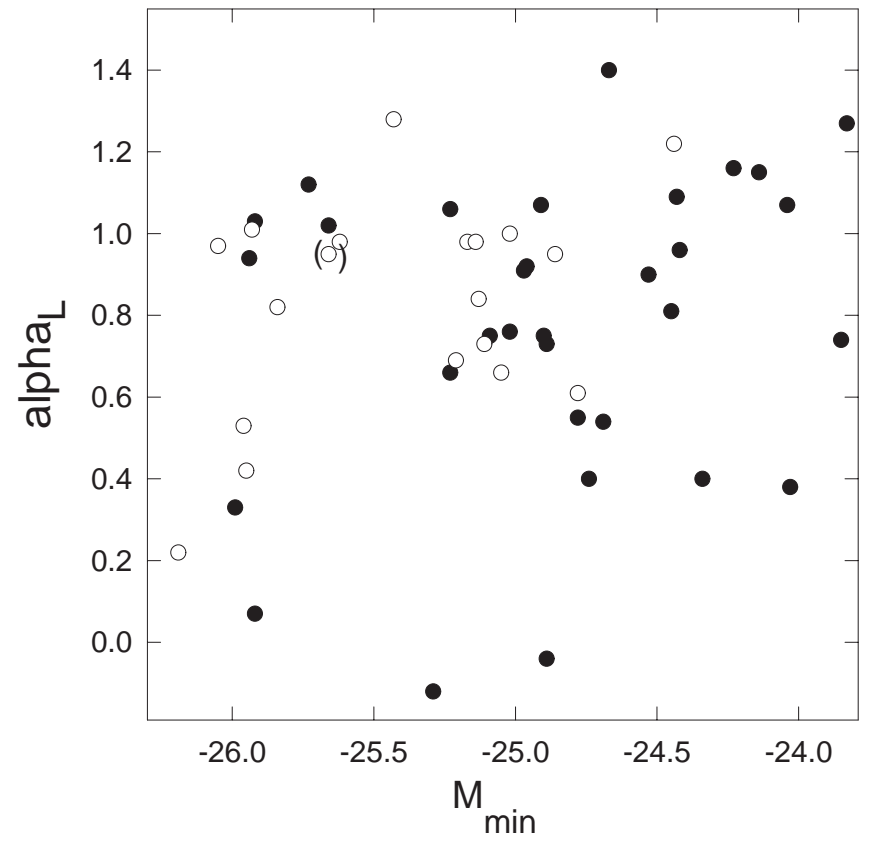

Fig. 8. Estimated lobe spectral index $\alpha_{\mathrm{L}}$ against $M_{\min }$. It was assumed in the calculation of $\alpha_{\mathrm{L}}$ that the core index $\alpha_{\mathrm{C}}=0.2$. Dots: $0.5<z<1.0$. Open circles: $1.0<z<1.65$.

\subsection{Double source morphology}

What happens to the morphology of the double sources when one crosses the optical gap? This was inspected from the maps for sources with LAS larger than about 10 arcsec.

The most telling difference in morphology, seen directly when comparing the maps, is that AI-sources have mostly a "dull" appearance of symmetric FRII sources with rather compact lobes, while the "edge" sources, at similar redshifts, show a more varied morphology, including secondary hotspots, hotspots in the trailing edge of one lobe, enhanced asymmetry and "dogleg" structure. The secondary hotspot or its remains (as discussed by Linfield \& Perley 1984 for galaxy 3C 111) tend to lie beyond the primary hotspot, detoriating the FRII image. Figure 9 shows that the sources in this edge magnitude range tend to lie below other quasars in the size vs. normalized core strenght diagram.

These differences are illustrated by Table 1 which gives, in the order of decreasing optical luminosity, the largest sources observed with good resolution and having the core-to-total flux ratio $<0.5$ : FRII is seen to dominate the "top-ten" AI's, while the "edge"-sources have often atypical features for $\mathrm{FRII}^{7}$.

Inspection of the Table shows that the morphological differences between $\mathrm{AI}$ and the edge sources are

\footnotetext{
7 Morphology references in Table 1: 1) Aldcroft et al. (1993), 2) Bogers et al. (1994), 3) Bridle et al. (1994), 4) Burns et al. (1984), 5) Feigelson et al. (1984), 6) Garrington et al. (1991), 7) Lonsdale et al. (1993), 8) Murphy et al. (1993), 9) Owen \& Puschell (1984), 10) Price et al. (1993), 11) Saikia (1984), 12) Saikia (1989), 13) Swarup et al. (1984), 14) Swarup et al. (1986), 15) Morganti et al. (1993), 16) Mantovani et al. (1992).
}

Table 1. Radio morphology for sources with $L A S>9^{\prime \prime}$.

\begin{tabular}{|c|c|c|c|}
\hline name & $z$ & $M$ & morphology \\
\hline $1206+4356$ & 1.400 & -26.05 & $\mathrm{HS} 2 \perp^{4,7}$ \\
\hline 0405-1219 & 0.574 & -25.99 & FRII,HS $2 \perp ?^{15}$ \\
\hline $2325+2920$ & 1.015 & -25.96 & FRII $^{6}$ \\
\hline $1602-0011$ & 1.625 & -25.95 & $\mathrm{FRII}^{2,7}$ \\
\hline 0350-0719 & 0.962 & -25.94 & FRII $^{14}$ \\
\hline $0710+1151$ & 0.768 & -25.92 & $\mathrm{FRII}^{2,3}$ \\
\hline $1055+2007$ & 1.110 & -25.84 & HS in trailing edge ${ }^{8}$ \\
\hline 0349-1438 & 0.614 & -25.73 & $\mathrm{FRII}^{2}$ \\
\hline $0856+1703$ & 1.449 & -25.66 & FRII $?^{14}$ \\
\hline $1356+5806$ & 1.371 & -25.62 & FRII $^{9}$ \\
\hline $1136-1334$ & 0.554 & -25.23 & very asymmetric $^{12}$ \\
\hline $0850+1403$ & 1.109 & -25.17 & HS in trailing edge ${ }^{4}$ \\
\hline $1511+1022$ & 1.546 & -25.11 & very asymmetric ${ }^{7}$ \\
\hline $0833+6254$ & 1.112 & -25.05 & HS in trailing edge ${ }^{9}$ \\
\hline $1137+6604$ & 0.646 & -24.96 & HS in trailing edge ${ }^{3}$ \\
\hline $1622+2352$ & 0.927 & -24.91 & HS in trailing edge $\mathrm{e}^{3,5}$ \\
\hline $0957+0019$ & 0.907 & -24.90 & HS in trailing edge $e^{4,10}$ \\
\hline 0454-2204 & 0.534 & -24.89 & FRII $^{1}$ \\
\hline $1509+1551$ & 0.828 & -24.89 & HS in trailing edge ${ }^{10}$ \\
\hline $1218+3359$ & 1.519 & -24.86 & "dogleg" structure ${ }^{6,7}$ \\
\hline $0922+1457$ & 0.896 & -24.74 & FRII HS $2 \perp^{13}$ \\
\hline $1327-2126$ & 0.528 & -24.69 & FRII mult.HS ${ }^{1}$ \\
\hline $1422+2013$ & 0.871 & -24.45 & $\mathrm{FRII}^{16}$ \\
\hline $1221+1837$ & 1.401 & -24.44 & FRII $?^{13}$ \\
\hline $1047+0941$ & 0.786 & -24.42 & FRII $^{10,11}$ \\
\hline $0838+1323$ & 0.684 & -24.34 & $\mathrm{FRII}^{2}$ \\
\hline $1305+0658$ & 0.602 & -24.23 & $\mathrm{FRII}^{2}$ \\
\hline $0932+0217$ & 0.659 & -24.04 & $\mathrm{FRII}^{2,10}$ \\
\hline $1634+2654$ & 0.561 & -23.83 & $\mathrm{FRII}^{2}$ \\
\hline $1618+1743$ & 0.555 & -23.71 & FRII HS $2 \perp^{3}$ \\
\hline
\end{tabular}

not connected with redshift. However, the sources fainter than -24.8 , which are typically FRII, have a significantly lower average $z$. We have included one quasar slightly fainter than the arbitrary limit $-23.8(1618+1743)$ in order to have 10 objects in each range. 


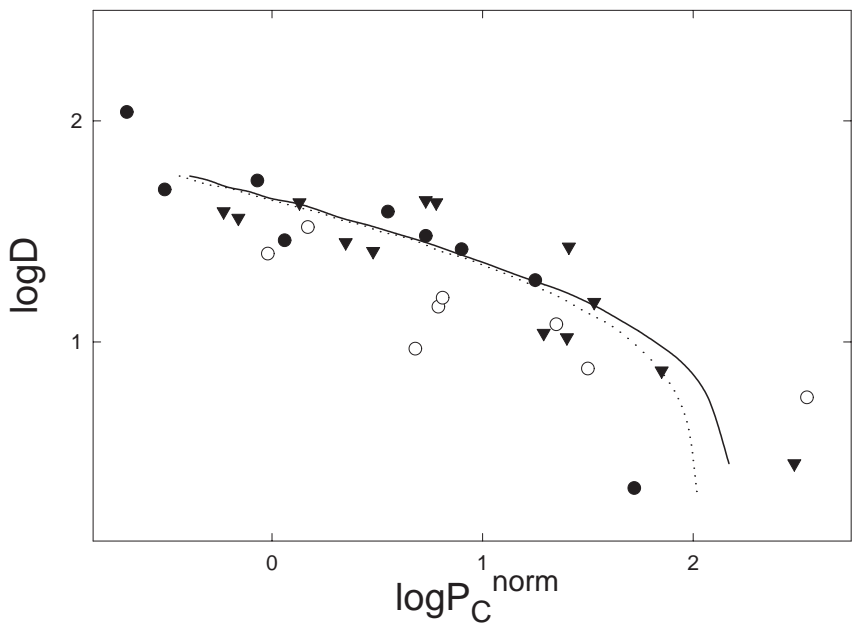

Fig. 9. Predicted projected size $\log D$ against boosted core power $\log P_{\mathrm{c}}$ for jet speed $\beta=0.993$ and viewing angle $\phi<30 \mathrm{deg}$ (upper curve), and for $\beta=0.983$ and $\phi<45 \mathrm{deg}$ (lower curve). The data, normalized to the same optical magnitude, are from Fig. 5a. Open circles are from the faint edge of the optical gap $(-25.5--24.8)$. Dots are AI, triangles from $(-24.8--23.8)$.

\section{Size vs. core power, Doppler boosting, and $\Gamma$}

To learn what is needed for the observed size-core power trend, calculations were made with the boosting formula giving the enhanced flux from the jet $S_{\mathrm{f}}$, or $S_{\mathrm{f}}=\delta^{p} S_{\mathrm{f}^{\prime}}$. Here $\delta=\left(1-\beta^{2}\right) /(1-\beta \cos \phi)$ and, for a continuous matter flow with speed $\beta, p=2+\alpha_{\mathrm{C}}$. $\phi$ is the viewing angle. The projected size $D$ is simply $\sin \phi D_{\text {o }}$.

The strong core enhancement requires a high $\beta$, similar to that needed for superluminal VLBI core phenomena and rapid radio variations. Figure 9 shows two theoretical curves, for $\beta=0.993$ and $\phi \leq 30 \mathrm{deg}$, and $\beta=0.983$ and $\phi \leq 45$. Such velocities give Lorentz factors $\Gamma=8$ and $=5$. However, a rather similar curve results if $\phi$ is allowed to go up to $90 \mathrm{deg}$, while $\beta$ is lowered to 0.975 $(\Gamma=4.5)$.

If the maximum viewing angle is $45 \mathrm{deg}$, the range of over 1 in $\log D$ implies that the minimum $\phi$ would reach $\approx 4$ deg or less. Lähteenmäki \& Valtaoja (1999) calculated the Lorentz factors and viewing angles for a few tens of core-dominated quasars and BLLac objects, which would populate the extreme right end of the size vs. core power diagram. They derived $\langle\Gamma\rangle \approx 7$ and $\langle\phi\rangle \approx 4 \mathrm{deg}$, in accordance with our interpretation of the $P_{\mathrm{C}}-D$ relation. In fact, Lähteenmäki \& Valtaoja have in their sample three of our sources $(1641+3954,2230+1128,2251+1552)$.

\section{Remarks and conclusions}

This paper has concentrated on the redshift range $0.5-1.65$ where one finds members of AI. We intend to study low $z$ quasars, when in future we compare double source quasars with radio galaxies at similar redshifts.
The "unified scheme" then will also be discussed (is there a radio galaxy subclass corresponding to the AI of quasars?).

We summarize the conclusions:

* AI double sources define the upper envelope in the size vs. redshift diagram, so these optically most luminous quasars are also in radio the largest sources in their redshift range;

* The quasars with shallow $\left(\alpha_{\text {lobe }}<0.7\right)$ or steep $\left(\alpha_{\text {lobe }}>1.0\right)$ spectra show a good correlation between projected size and core power, similar to what is expected from different orientations and the Doppler boosting of the core, with the Lorenz factor $\Gamma \approx 5-8$. AI define the upper envelope;

* The quasars with "non-Kardashev" intermediate spectral index do not show any correlation in the core power-size diagram. These sources often deviate morphologically from good FRII, have enhanced $Q$ asymmetry, and their bending angle does not converge towards a small value for large sizes;

* The AI double sources are rather symmetric and mostly have a typical FRII structure. The quasars within a narrow $(0.5-0.8 \mathrm{mag})$ strip on the fainter side of the optical gap seem to be more asymmetric and to deviate from good FRII. They often have a hotspot in the trailing edge of a lobe.

Acknowledgements. The valuable comments by the patient referee are gratefully acknowledged. I also thank Boris Komberg and Kari Nilsson for useful discussions. This study has been supported by the Academy of Finland (project "Cosmology from the local to the deep galaxy universe").

\section{Appendix A: Data for double radio sources}

Table A.1 gives data for known double sources among the quasars used in Paper I, with $-26.5<M_{\min }<-23.8$. The radio data come from Nilsson (1998).

1,2) coordinates $\alpha, \delta$;

3) redshift $z$;

4) absolute minimum brightness $V$ magnitude, $M_{\min }$;

5) largest angular size LAS;

6) flux at $5 \mathrm{GHz}, S 5$;

7) core flux at $5 \mathrm{GHz}, S 5_{\mathrm{C}}$ (asterix: from Hintzen et al. $19831.4 \mathrm{GHz}$ value, with $\left.\alpha_{\mathrm{c}}=0.2\right)$;

8) bending angle $\Phi$;

9) spectral index from $2.7 \mathrm{GHz}$ to $5 \mathrm{GHz}, \alpha_{2.7-5.0}$;

10) lobe-core distance asymmetry, $Q$;

11) lobe flux asymmetry, $f_{\mathrm{r}}(<1$ for the Mackay rule);

12) derived lobe spectral index $\alpha_{\mathrm{L}}$;

13) $(\log )$ linear size $\log D$;

14) (log) core power $\log P_{\mathrm{C}}$. 
Table A.1. Double radio sources $\left(M_{\min }<-23.8 \mathrm{mag}\right)$.

\begin{tabular}{|c|c|c|c|c|c|c|c|c|c|c|c|c|c|}
\hline $\mathrm{RA}$ & $\delta$ & $z$ & $M$ & $L A S$ & $S 5$ & $S 5_{\mathrm{C}}$ & $\Phi$ & $\alpha_{2.7-5.0}$ & $Q$ & $f_{\mathrm{r}}$ & $\alpha_{\mathrm{L}}$ & $\log D$ & $\log P_{\mathrm{C}}$ \\
\hline 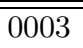 & $\overline{c+1553}$ & 0.450 & -24.52 & 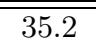 & 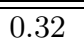 & 125.0 & $\overline{88}$ & 0.59 & 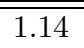 & 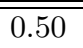 & 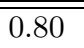 & 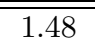 & 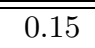 \\
\hline 0155 & -1058 & 0.616 & -24.53 & 4.3 & 0.75 & - & - & 0.90 & - & - & 0.90 & 0.62 & - \\
\hline 0159 & -1147 & 0.669 & -24.43 & 15.4 & 1.37 & 845.0 & 23 & 0.60 & 9.28 & - & 1.09 & 1.18 & 1.30 \\
\hline 0349 & -1438 & 0.614 & -25.73 & 114.0 & 0.59 & 20.0 & 7 & 1.10 & 1.06 & 1.64 & 1.12 & 2.04 & -0.40 \\
\hline 0350 & -0719 & 0.962 & -25.94 & 42.5 & 0.79 & 12.0 & 4 & 0.93 & 1.51 & 9.65 & 0.94 & 1.65 & -0.26 \\
\hline 0405 & -1219 & 0.574 & -25.99 & 32.0 & 1.83 & 760.0 & 11 & 0.28 & 1.39 & - & 0.33 & 1.48 & 1.13 \\
\hline 0454 & -2204 & 0.534 & -24.89 & 87.0 & 1.10 & 140.0 & 0 & 0.67 & 1.32 & 1.54 & 0.73 & 1.90 & 0.34 \\
\hline 0710 & +1151 & 0.768 & -25.92 & 48.0 & 0.66 & 23.5 & 12 & 1.01 & 1.34 & 0.77 & 1.03 & 1.69 & -0.15 \\
\hline 0725 & +1443 & 1.382 & -25.02 & 5.9 & 0.66 & 6.0 & 3 & 0.99 & 2.69 & 2.03 & 1.00 & 0.80 & -0.28 \\
\hline 0740 & +3800 & 1.063 & -25.21 & 1.6 & 0.38 & - & 5 & 0.69 & - & 1.00 & 0.69 & 0.23 & - \\
\hline 0742 & +3150 & 0.462 & -25.40 & 115.0 & 0.98 & 640.0 & 4 & 0.21 & - & 0.86 & 0.23 & 1.99 & 0.88 \\
\hline 0809 & +4822 & 0.871 & -24.97 & 5.4 & 4.36 & 6.0 & 6 & 0.91 & 1.99 & 1.25 & 0.91 & 0.75 & -0.64 \\
\hline 0833 & +6524 & 1.112 & -25.05 & 31.0 & 0.34 & 26.1 & 4 & 0.63 & 1.42 & 0.75 & 0.66 & 1.52 & 0.19 \\
\hline 0838 & +1323 & 0.684 & -24.34 & 11.2 & 1.44 & 435.0 & 21 & 0.34 & 1.20 & 1.89 & 0.40 & 1.04 & 1.03 \\
\hline 0850 & +1403 & 1.109 & -25.17 & 11.0 & 0.54 & 51.0 & 2 & 0.92 & 1.32 & 3.45 & 0.98 & 1.07 & 0.48 \\
\hline 0855 & +1421 & 1.048 & -25.16 & 9.0 & 0.89 & 310.0 & 0 & 0.75 & 1.05 & 0.43 & 0.98 & 0.98 & 1.22 \\
\hline 0856 & +1703 & 1.449 & -25.66 & 10.3 & 0.19 & 10.0 & 29 & 0.92 & 1.65 & 3.71 & 0.95 & 1.04 & -0.02 \\
\hline 0906 & +4305 & 0.670 & -23.85 & 1.6 & 1.81 & 1050.0 & 26 & 0.45 & 1.25 & 0.67 & 0.74 & 0.20 & 1.39 \\
\hline 0922 & +1457 & 0.896 & -24.74 & 41.1 & 0.18 & 26.0 & 1 & 0.37 & 1.23 & 1.36 & 0.40 & 1.63 & 0.02 \\
\hline 0932 & +0217 & 0.659 & -24.04 & 44.8 & 0.28 & 97.0 & 9 & 0.82 & 6.00 & 1.94 & 1.07 & 1.64 & 0.35 \\
\hline 0957 & +0019 & 0.907 & -24.90 & 31.4 & 0.33 & 93.1 & 21 & 0.61 & 1.39 & 1.30 & 0.75 & 1.52 & 0.58 \\
\hline 1004 & +1303 & 0.240 & -24.56 & 115.0 & 0.47 & 12.0 & 1 & 0.68 & 1.49 & 1.46 & 0.69 & 1.84 & -1.39 \\
\hline 1022 & +1927 & 0.828 & -24.90 & 11.5 & 0.74 & 581.0 & 0 & -0.50 & 1.68 & - & - & 1.08 & 1.31 \\
\hline 1040 & +1219 & 1.029 & -25.43 & 7.1 & 1.39 & 910.0 & 2 & 0.66 & 1.69 & 3.22 & 1.28 & 0.88 & 1.67 \\
\hline 1047 & +0941 & 0.786 & -24.42 & 21.1 & 0.16 & 29.0 & 7 & 0.85 & 2.14 & 0.15 & 0.96 & 1.33 & -0.04 \\
\hline 1048 & -0902 & 0.344 & -23.76 & 83.2 & 0.51 & 33.0 & 2 & 0.92 & 1.29 & 1.13 & 0.96 & 1.79 & -0.65 \\
\hline 1049 & +6141 & 0.422 & -23.89 & 2.5 & 0.38 & - & - & - & - & - & - & 0.31 & - \\
\hline 1055 & +2007 & 1.110 & -25.84 & 46.4 & 1.68 & 560.1 & 37 & 0.64 & 1.31 & 0.16 & 0.82 & 1.70 & 1.52 \\
\hline 1100 & +7715 & 0.311 & -24.35 & 23.0 & 0.78 & 71.0 & 9 & 0.95 & 2.01 & 1.83 & 1.01 & 1.21 & -0.40 \\
\hline 1103 & -0036 & 0.426 & -24.14 & 22.3 & 0.54 & 121.0 & 1 & 0.78 & 1.21 & 1.18 & 0.91 & 1.27 & 0.09 \\
\hline 1104 & +1644 & 0.634 & -25.92 & 40.0 & 0.63 & 388.0 & 3 & 0.15 & 1.01 & 0.54 & 0.07 & 1.59 & 0.92 \\
\hline 1136 & -1334 & 0.554 & -25.23 & 15.8 & 2.29 & 461.0 & 5 & 0.58 & 2.21 & 1.21 & 0.66 & 1.16 & 0.88 \\
\hline 1137 & +6604 & 0.646 & -24.96 & 48.0 & 1.04 & 157.0 & 0 & 0.83 & 1.94 & 0.25 & 0.92 & 1.67 & 0.54 \\
\hline 1148 & +3842 & 1.303 & -25.89 & 7.8 & 0.21 & 343.0 & 23 & 0.76 & 1.09 & 0.83 & - & 0.92 & 1.43 \\
\hline 1150 & +4947 & 0.334 & -23.87 & 16.0 & 0.70 & 310.0 & 4 & 0.57 & 1.02 & 0.85 & 0.81 & 1.07 & 0.29 \\
\hline 1150 & +0930 & 0.698 & -24.14 & 5.3 & 0.50 & - & - & - & - & - & - & 0.72 & - \\
\hline 1153 & +3144 & 1.557 & -25.13 & 0.9 & 1.15 & - & 3 & 0.84 & 2.00 & 0.48 & 0.84 & -0.02 & - \\
\hline 1206 & +4356 & 1.400 & -26.05 & 9.8 & 0.60 & 50.0 & 14 & 0.92 & 1.17 & 0.19 & 0.97 & 1.02 & 0.65 \\
\hline 1208 & +3213 & 0.388 & -24.58 & 82.0 & 0.11 & 43.0 & 2 & 0.32 & 1.50 & 0.18 & 0.39 & 1.81 & -0.44 \\
\hline 1218 & +3359 & 1.519 & -24.86 & 9.2 & 0.87 & 116.0 & 36 & 0.87 & 1.23 & 0.22 & 0.95 & 0.99 & 1.08 \\
\hline 1221 & +1837 & 1.401 & -24.44 & 24.0 & 0.15 & 20.0 & 15 & 1.12 & 1.56 & 0.81 & 1.22 & 1.41 & 0.25 \\
\hline 1229 & -0207 & 1.038 & -25.93 & 18.0 & 1.10 & $795.0^{*}$ & 0 & 0.47 & 2.66 & 0.45 & 1.01 & 1.28 & 1.62 \\
\hline 1232 & -2455 & 0.355 & -24.38 & 86.0 & 0.54 & 11.0 & 6 & 0.80 & 1.71 & 1.28 & 0.81 & 1.81 & -1.10 \\
\hline 1302 & -1017 & 0.286 & -24.23 & 15.0 & - & 1185.0 & 61 & -0.06 & 1.64 & - & - & 1.00 & 0.75 \\
\hline 1305 & +0658 & 0.602 & -24.23 & 41.0 & 0.31 & 15.0 & 2 & 1.12 & 1.73 & 0.62 & 1.16 & 1.59 & -0.54 \\
\hline 1327 & -2126 & 0.528 & -24.69 & 31.0 & 0.51 & 110.0 & 15 & 0.47 & 1.23 & 1.62 & 0.54 & 1.45 & 0.22 \\
\hline 1328 & +3045 & 0.849 & -24.67 & 2.7 & 7.48 & 6100.0 & 35 & 0.51 & 3.37 & 1.54 & 1.40 & 0.45 & 2.35 \\
\hline 1340 & +6036 & 0.961 & -24.14 & 7.0 & 0.40 & - & 17 & 1.15 & 1.15 & 0.43 & 1.15 & 0.87 & - \\
\hline 1354 & +1933 & 0.720 & -25.05 & 43.7 & 1.76 & 2016.0 & 7 & 0.24 & 1.80 & 1.04 & - & 1.64 & 1.74 \\
\hline 1356 & +5806 & 1.371 & -25.62 & 45.0 & 0.16 & 7.7 & 1 & 0.95 & 1.17 & - & 0.98 & 1.68 & -0.18 \\
\hline 1415 & +1717 & 0.821 & -25.09 & 7.9 & 0.28 & 18.0 & 84 & 0.72 & 1.58 & 2.26 & 0.75 & 0.91 & -0.21 \\
\hline 1416 & +1554 & 1.472 & -26.19 & 27.1 & 0.08 & $35.0^{*}$ & 2 & 0.21 & 1.38 & 0.46 & 0.22 & 1.46 & 0.54 \\
\hline 1422 & +2013 & 0.871 & -24.45 & 11.0 & 0.62 & 33.0 & 10 & 0.78 & 2.65 & 2.44 & 0.81 & 1.06 & 0.10 \\
\hline 1425 & +2645 & 0.366 & -25.05 & 240.0 & 0.11 & 46.1 & 10 & 1.22 & 1.71 & 0.30 & 1.69 & 2.26 & -0.46 \\
\hline 1433 & +1742 & 1.203 & -24.78 & 9.7 & 0.56 & 302.0 & 19 & 0.40 & 1.16 & 0.11 & 0.61 & 1.02 & 1.32 \\
\hline 1453 & -1056 & 0.938 & -25.02 & 41.0 & 1.55 & - & 17 & 0.76 & 2.50 & 3.24 & 0.76 & 1.64 & - \\
\hline
\end{tabular}


Table A.1. continued.

\begin{tabular}{cccccccccccccc}
\hline \hline RA & $\delta$ & $z$ & $M$ & $L A S$ & $S 5$ & $S 5_{\mathrm{C}}$ & $\Phi$ & $\alpha_{2.7-5.0}$ & $Q$ & $f_{\mathrm{r}}$ & $\alpha_{\mathrm{L}}$ & $\log D$ & $\log P_{\mathrm{C}}$ \\
\hline \hline 1458 & +7152 & 0.905 & -25.66 & 2.1 & 3.76 & 2350.0 & 37 & 0.56 & 1.38 & 2.67 & 1.02 & 0.34 & 1.98 \\
1509 & +1551 & 0.828 & -24.89 & 9.1 & 0.45 & 125.0 & 48 & 0.03 & 3.90 & 2.36 & -0.04 & 0.97 & 0.64 \\
1511 & +1022 & 1.546 & -25.11 & 33.0 & 0.07 & 11.9 & 21 & 0.65 & 1.42 & 10.00 & 0.73 & 1.55 & 0.10 \\
1512 & +3701 & 0.370 & -24.13 & 58.4 & 0.33 & 80.0 & 4 & 0.14 & 1.22 & 0.82 & 0.12 & 1.65 & -0.21 \\
1548 & +1129 & 0.436 & -24.11 & 45.0 & 0.54 & 353.0 & 56 & 0.39 & 1.15 & 0.42 & 0.69 & 1.58 & 0.57 \\
1602 & -0011 & 1.625 & -25.95 & 25.0 & 0.38 & 165.0 & 9 & 0.33 & 2.44 & 1.60 & 0.42 & 1.42 & 1.28 \\
1618 & +1743 & 0.555 & -23.71 & 46.0 & 0.57 & 111.0 & 3 & 1.36 & 1.64 & 1.40 & 1.55 & 1.63 & 0.27 \\
1622 & +2352 & 0.927 & -24.91 & 24.0 & 0.69 & 20.4 & 13 & 1.05 & 1.63 & 1.85 & 1.07 & 1.40 & -0.06 \\
1634 & +2654 & 0.561 & -23.83 & 39.0 & 0.43 & 14.0 & 3 & 1.24 & 1.38 & 0.19 & 1.27 & 1.56 & -0.62 \\
1641 & +3954 & 0.594 & -24.55 & 2.8 & 5.50 & 8610.0 & - & 0.10 & - & - & - & 0.42 & 2.21 \\
1704 & +6048 & 0.371 & -24.79 & 64.0 & 1.21 & 6.5 & 5 & 0.84 & 1.36 & 0.07 & 0.84 & 1.69 & -1.30 \\
1725 & +0429 & 0.296 & -24.48 & 4.4 & 0.80 & 730.0 & - & -0.71 & - & - & - & 0.48 & 0.57 \\
1828 & +4842 & 0.692 & -24.78 & 7.5 & 7.50 & 2300.0 & - & 0.45 & - & - & 0.55 & 0.87 & 1.76 \\
1830 & +2831 & 0.594 & -24.03 & 28.2 & 1.07 & 551.0 & 18 & 0.29 & 1.02 & 0.60 & 0.38 & 1.43 & 1.02 \\
2115 & -3031 & 0.980 & -25.23 & 15.0 & 0.73 & 170.0 & 25 & 0.90 & 1.00 & 4.09 & 1.06 & 1.20 & 0.91 \\
2201 & +3131 & 0.298 & -25.36 & 80.0 & 3.16 & 1500.0 & 13 & -0.27 & 1.14 & - & -0.85 & 1.74 & 0.88 \\
2230 & +1128 & 1.037 & -24.83 & 2.4 & 3.77 & 3740.0 & 5 & 0.49 & 2.00 & 1.82 & - & 0.41 & 2.29 \\
2251 & +1552 & 0.859 & -25.29 & 5.4 & 18.20 & 12200.0 & - & 0.10 & - & - & -0.12 & 0.75 & 2.66 \\
2251 & +1120 & 0.323 & -24.81 & 9.8 & 0.58 & 21.0 & 3 & 0.68 & 1.71 & 0.52 & 0.70 & 0.85 & -0.90 \\
2308 & +0951 & 0.432 & -25.05 & 82.0 & 0.26 & 102.0 & 8 & 0.41 & 1.05 & 0.40 & 0.53 & 1.83 & 0.03 \\
2325 & +2920 & 1.015 & -25.96 & 51.0 & 0.38 & 41.2 & 0 & 0.50 & 1.18 & 0.67 & 0.53 & 1.73 & 0.32 \\
\hline
\end{tabular}

\section{References}

Aldcroft, T. L., Elvis, M., \& Bechtold, J. 1993, AJ, 105, 2054 Barthel, P. D., \& Miley, G. K. 1988, Nature, 333, 319

Barthel, P. D., Vestergaard, M., \& Lonsdale, C. J. 2000, A\&A, 354,7

Baryshev, Yu., \& Teerikorpi, P. 1995, A\&A, 295, 11

Bogers, W. J., Hes, R., Barthel, P. D., \& Zensus, J. A. 1994, A\&AS, 105, 91

Bridle, A. H., Hough, D. H., Lonsdale, C. J., Burns, J. O., \& Laing, R. A. 1994, AJ, 108, 766

Burns, J. O., Basart, J. P., de Young, D. S., \& Ghiglia, D. C. 1984, ApJ, 283, 515

Feigelson, E. D., Isobe, T., \& Kembhavi, A. 1984, AJ, 89, 1464

Garrington, S. T., Conway, R. G., \& Leahy, J. P. 1991, MNRAS, 250, 171

Hintzen, P., Ulvestad, J., \& Owen, F. 1983, AJ, 88, 709

Kardashev, N. S. 1962, SvA, 6, 317

Linfield, R., \& Perley, R. 1984, ApJ, 279, 60

Lister, M. L., Gower, A. C., \& Hutchings, J. B. 1994, AJ, 108, 821

Lonsdale, C. J., Barthel, P. D., \& Miley, G. K. 1993, ApJS, 87,63

La̋hteenma̋ki, A., \& Valtaoja, E. 1999, ApJ, 521, 493

Mack, C. 1975, Essentials of Statistics for Scientists and Technologists (Plenum Press, New York)

Mackay, C. D., 1971, MNRAS, 154, 209

Mantovani, F., Junor, W., Fanti, R., et al. 1992, MNRAS, 257, 353
Meisenheimer, K., Rser, H.-J., Hiltner, P. R., et al. 1989, A\&A, 219,63

Morganti, R., Killeen, N. E. B., \& Tadhunter, C. N. 1993, MNRAS, 263, 1023

Murphy, D. W., Browne, I. W. A., \& Perley, R. A. 1993, MNRAS, 264, 298

Nilsson, K. 1998, A\&AS, 132, 31

Orr, M. J. L., \& Browne, I. W. A. 1982, MNRAS, 200, 1067

Owen, F. N., \& Puschell, J. J. 1984, AJ, 89, 932

Price, R., Gower, A. C., Hutchings, J. B., et al. 1993, ApJS, 86,365

Riley, J. M., \& Jenkins, C. J. 1977, IAU Symp., 74, 237

Saikia, D. J., Hastri, P., Sinha, R. P., Kapahi, V. K., \& Swarup, G. 1984, JA\&A, 5, 429

Saikia, D. J., Shastri, P., Cornwell, T. J., Junor, W., \& Muxlow, T. W. B. 1989, JA\&A, 10, 203

Siegel, S. 1956, Non-parametric statistics for the behavioral scientist (McGraw-Hill, New York)

Swarup, G., Sinha, R. P., \& Hilldrup, K. 1984, MNRAS, 208, 813

Swarup, G., Saikia, D. J., Beltrametti, M., Sinha, R. P., \& Salter, C. J. 1986, MNRAS, 220, 1

Teerikorpi, P. 1981, A\&A, 98, 309, Paper I

Teerikorpi, P. 2000, A\&A, 353, 77, Paper II

Valtaoja, E., Teräsranta, H., Urpo, S., et al. 1988, A\&A, 254, 71

Wills, B. J., \& Brotherton, M. S. 1995, ApJ, 448, L81 